\title{
A "Needling" Problem: Shoulder Injury Related to Vaccine Administration
}

\author{
Matthew G. Barnes, MD, Christopher Ledford, MD, and Karen Hogan, BA
}

A 22-year-old woman with no significant medical history developed acute left shoulder pain and severe restrictions in range of motion after a seasonal influenza vaccine injection. Imaging by MRI, approximately 8 weeks after the injection, and by ultrasound, approximately 9.5 weeks after the injection demonstrated contusions on the humerus, injury of the supraspinatus, and effusion in the subacromial bursa. Her reaction was reported to the Vaccine Adverse Event Reporting System as a case of shoulder injury related to vaccine administration, likely due to injection of the influenza vaccine into the subacromial bursa. This case serves as a catalyst for discussion regarding vaccination technique and the potential to prevent complications arising from vaccine overpenetration. (J Am Board Fam Med 2012; 25:919-922.)

Keywords: Arthritis, Case Reports, Immunizations, Immunology, Shoulder, SIRVA, Sports Medicine, Vaccination

\section{Description}

Signs of adhesive capsulitis and a rotator cuff tear with cortical irregularity 6 months after a routine seasonal flu injection were the symptoms of shoulder injury related to vaccine administration.

\section{Case History}

A 22-year-old woman with a weight of 120 lbs (54 $\mathrm{kg}$ ) and no significant prior medical history presented with acute left shoulder pain, which limited her range of motion and functional ability. The patient was in her usual state of health until she received the influenza vaccine approximately 2 months before initial presentation. The 2011 seasonal flu vaccine was given by a trained medical technician, who reported placing the vaccine "3 fingers from the acromion" (measured at 2.75 inches or approximately $7 \mathrm{~cm}$ ) in the middle of the deltoid, "hubbing" (inserting the needle to the

\footnotetext{
This article was externally peer reviewed.

Submitted 13 December 2011; revised 5 April 2012; accepted 23 April 2012.

From the Dewitt Army Community Hospital, Ft. Belvoir, VA (MGB, CL, KH); and the U.S. Air Force Wilford Hall Medical Center, San Antonio, TX (MGB).

Funding: none.

Conflict of interest: none declared.

Corresponding author: Matthew G. Barnes, MD, Wilford Hall Medical Center, Family Health Clinic, 2200 Bergquist Drive, San Antonio, TX 78236.
}

depth of the plastic hub but not depressing surrounding skin) a 1-inch, 23-gauge needle, and injecting the vaccine. The patient reported having the onset of shoulder pain at the administration site within 2 hours of vaccination. Over the next 6 weeks, she had progressive limitations of her range of motion of her left shoulder and increasing pain, 9 out of 10 pain on a pain scale. On examination, the patient had very limited range of motion and experienced pain with both active and passive range of motion. Plain radiographs were unremarkable. Ultrasound imaging, at 9.5 weeks after the initial injection, demonstrated a partial tear of the supraspinatus tendon as well as cortical irregularity on the superolateral humeral head at the point of injection (Figure 1). MRI, at 8 weeks after the initial injection, showed joint effusion in the subacromial bursa, a longitudinal partial tear of the supraspinatus tendon, and a consistent bony contusion (Figure 2). Due to her limited range of motion, the patient had an immediate referral to sports medicine and was referred for aggressive physical therapy. A report of this patient's history was sent to the Vaccine Adverse Event Reporting System.

At 11 weeks after initial presentation, the patient was re-evaluated and found to have significant improvement in pain (1 out of 10$)$ at rest, reporting discomfort with use of the left shoulder 
Figure 1. Ultrasound image of shoulder, 9.5 weeks after vaccination. Lower arrow indicates cortical irregularity on the superolateral humeral head. Upper arrow indicates partial tear of the supraspinatus tendon.

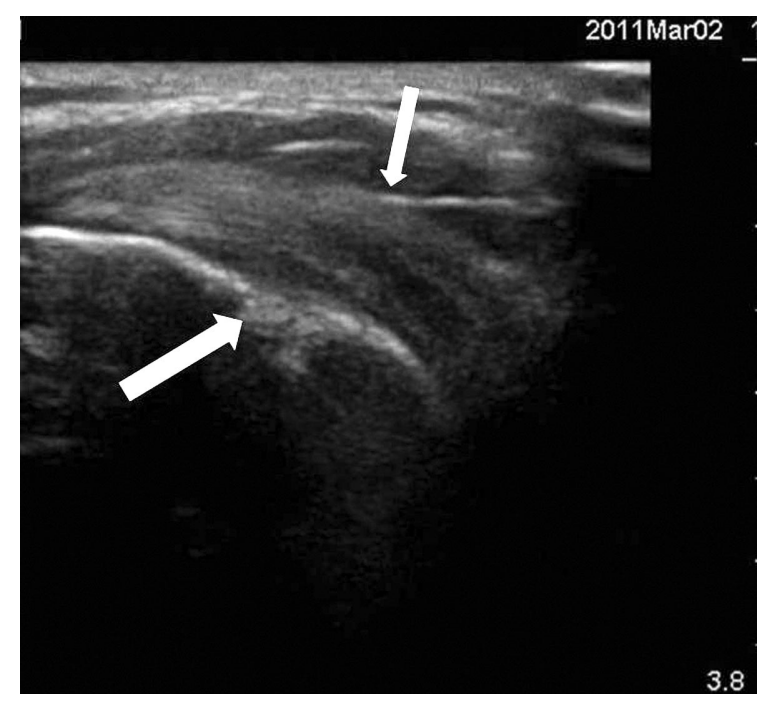

but no limitations in function. Ultrasound images at that time demonstrated residual fluid at the supraspinatus with a consistent cortical irregularity. In follow-up with the patient, at 16 months after the initial injection, she reports that her range of motion and strength continue to allow her to conduct all her desired daily activities.

\section{Literature Search}

A PubMed search was performed in March 2012, in preparation for publication, using the term "Shoulder Injury Related to Vaccine Administration” for all time periods and limited to English language publications, which returned a single article. ${ }^{1}$ The article was obtained and its 15 citations were reviewed for pertinence yielding 6 citations. These 6 articles then had their citations reviewed, which led to 2 more relevant articles, neither of which identified additional pertinent citations. The search terms were broadened for "vaccine related shoulder dysfunction," which only returned the article which prompted the term's search.

\section{Discussion}

A search of Vaccine Adverse Event Reporting System data from 2010 to 2012 for adverse events that included influenza vaccination and joint complaints revealed 1068 entries. When further limited to the word "shoulder" in the adverse event description, there were 40 males (3.7\%) and 127 females $(11.9 \%)$ that made up the reports. ${ }^{2}$ There is no way to be certain of the total number of influenza immunizations given during this time period; however, a conservative estimated from the Centers for Disease Control and Prevention surveying mechanism reveals that approximately $43 \%$ of the US population (age $>6$ months) got seasonal influenza immunization in the 2010 to 2011 season. $^{3}$ The relatively small numbers of adverse reactions reinforces the concept that adverse reactions, specifically those involving the shoulder, are very rare. The purpose of this case report is not to further vaccine hesitancy but to broaden differential diagnoses when shoulder pain presents in the clinic in the context of prior immunization.

This patient developed signs and symptoms consistent with previously described cases of shoulder injury related to vaccine administration (SIRVA) in the literature. The patient was female, previously exposed to the influenza vaccine, and developed multiple shoulder injuries almost immediately after vaccine administration. A case series from Atanasoff described 13 cases, of which 11 were female ( $85 \%$ of the cases); 8 involved influenza vaccine $(62 \%)$ and 12 involved onset of signs and symptoms

Figure 2. T2-weighted MRI image of shoulder, 8 weeks after vaccination. Lower left arrow indicates humeral head cortical irregularity. Upper middle arrow indicates partial tear of supraspinatus tendon. Upper right arrow indicates effusion at subacromial bursa.

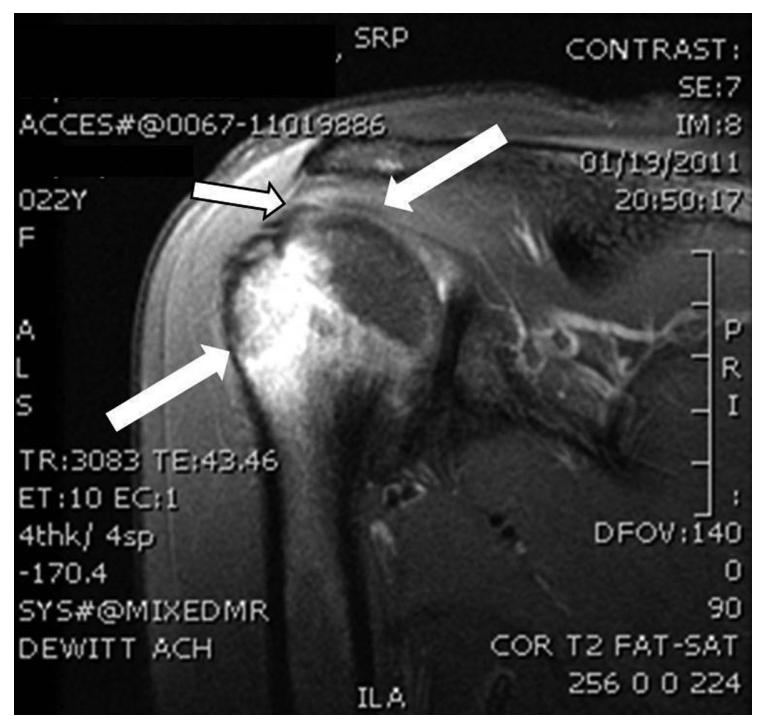


immediately or within 24 hours of administration. ${ }^{1}$ This case series reported that average body mass index was $27.2 \mathrm{~kg} / \mathrm{m}^{2}$. A case series by Bodor ${ }^{4}$ described 2 similar cases: 1 occurring after seasonal flu vaccine administration and 1 occurring after polyvalent pneumonia vaccine administration; this case series did not comment on the body mass index or body habitus of the cases. A review of the literature demonstrates a lack of data on SIRVA. There have only been 2 studies and a total case load of 15 patients making this a rare vaccination-related injury. However, cases are likely underreported, leading to an unknown incidence and a lack of literature.

The authors of these case series suggested that SIRVA is due to an inflammatory effect from vaccine administration into the subdeltoid bursa. This inflammatory response may be due either to the antigenic or nonantigenic components of the vaccine (antimicrobial, preservative, etc). They based these assumptions on the consistent presentations of adhesive capsulitis (as demonstrated by the patient's pain, lack of motion, weakness, and impaired mobility/functionality) and multiple noted pathologies on imaging, more than would be expected by the trauma of vaccination. Previously, there was only a single documented case of bone friability in SIRVA. ${ }^{1}$ This current case has a previously unreported finding of persistent cortical irregularity, making a stronger case for the inflammatory response of the vaccine as the pathogenic factor of SIRVA.

Although there have been numerous studies discussing the potential dangers of underpenetration at the time of administering vaccines (including skin reaction and possible decreased immunogenicity), there is a surprising lack of data about side effects from overpenetration. Current Centers for Disease Control and Prevention guidelines state that a 1-inch needle depth is appropriate for most patients (except for persons $>200 \mathrm{lbs}$ or newborns), 5,9 which is a seemingly "one-size-fits-all" approach for an increasingly diverse population. A study of MRI deltoid fat pads by Lippert found that with using 5/8-inch, 7/8-inch, or 1-inch needles for intramuscular deltoid injections would cause $11 \%$ (16 of 150 ), $55 \%$ (83 of 150), and 61\% (92 of 150) of patients to experience overpenetration, respectively. $^{6}$

Based on Lippert's study, corroborated by an ultrasonographic study of deltoids by Koster ${ }^{7}$ and
Poland, ${ }^{8}$ a compelling argument could be made to change vaccination technique. Lippert suggests a weight-based vaccination technique: for instance, that a $1 / 2$-inch needle be used for any female who weighs up to $70 \mathrm{~kg}$ and any male who weighs up to $75 \mathrm{~kg}$. A 5/8-inch needle is recommended for any female who weighs between 70 and $115 \mathrm{~kg}$ and any male who weighs between 75 and $140 \mathrm{~kg}$. A 7/8inch or longer needle should be used for any female who weighs $115 \mathrm{~kg}$ and any male who weighs 140 $\mathrm{kg}$. This recommendation could conceivably create a $0 \%$ overpenetration rate and a $10 \%$ underpenetration rate. The patient in this case, at a weight of $120 \mathrm{lbs}(54 \mathrm{~kg})$, would have had a $1 / 2$-inch needle length, half the size of the needle recommended. These methods could potentially prevent SIRVA as well as other problems associated with overpenetration of vaccination.

\section{Conclusions}

Consider SIRVA as part of the differential diagnosis in patients presenting with acute shoulder pain after vaccine administration. This case serves as a reminder for what may be an underreported condition. This underreporting may be due, in part, to the Vaccine Adverse Event Reporting System being a passive reporting mechanism or to concerns for medicolegal implications for reporting. Additionally, the vaccine over penetration that likely led to SIRVA could have been prevented by refining the Centers for Disease Control and Prevention's current injection guidelines.

\section{References}

1. Atanasoff S, Ryan T, Lightfoot R, Johann-Liang R. Shoulder injury related to vaccine administration (SIRVA). Vaccine. 2010;28:8049-52.

2. Vaccine Adverse Event Reporting System. Available at: www.Vaers.hhs.gov/index. Accessed April 1, 2012.

3. Final state-level influenza vaccination coverage estimates for the 2010-11 season-United States, National Immunization Survey and Behavioral Risk Factor Surveillance System, August 2010 through May 2011. Available at: www.cdc.gov/flu/professionals/ vaccination/coverage_1011estimates.htm. Accessed April 1, 2012.

4. Bodor M, Motalvo E. Vaccination-related shoulder dysfunction. Vaccine. 2007;25:585-7.

5. Centers for Disease Control and Prevention. Dose, route, site, and needle size. Available at: www.immunize. org/catg.d/p3085.pdf. Accessed April 15, 2011. 
6. Lippert WC, Wall EJ. Optimal intramuscular needlepenetration depth. Pediatrics 2008;122:e556-63.

7. Koster MP, Stellato N, Kohn N, Rubin LG. Needle length for immunization of early adolescents as determined by ultrasound. Pediatrics 2009;124:667-72.

8. Poland GA, Borrud A, Jacobson RM, McDermott K, Wollan PC, Brakke D, et al. Determination of deltoid fat pad thickness. Implications for needle length in adult immunization. JAMA 1997;277:1709-11.

9. National Center for Immunization and Respiratory Diseases. General recommendations on immunization. Recommendations of the Advisory Committee on Immunization Practices (ACIP). MMWR Recomm Rep 2011;60:1-64. 